\title{
MR Spectroscopy of the Fetal Brain: Is It Possible without Sedation?
}

\author{
V. Berger-Kulemann, P.C. Brugger, D. Pugash, M. Krssak, M. Weber, A. Wielandner, and D. Prayer
}

\begin{abstract}
BACKGROUND AND PURPOSE: The quality of spectroscopic studies may be limited because of unrestricted fetal movement. Sedation is recommended to avoid motion artefacts. However, sedation involves side effects. The aim of this study was to assess the feasibility and quality of brain ${ }^{1} \mathrm{H}-\mathrm{MR}$ spectroscopy in unsedated fetuses and to evaluate whether quality is dependent on the type of spectra, fetal presentation, GA, and/or fetal pathology.
\end{abstract}

MATERIALS AND METHODS: Seventy-five single-voxel spectroscopic studies of the fetal brain, performed at gestational weeks 19-38 at $1.5 \mathrm{~T}$, were evaluated retrospectively. A PRESS (TE $=144$ or $35 \mathrm{~ms}$ ) was used. Fetal presentation, $\mathrm{GA}$, and kind of pathology were recorded. The quality of the spectra was assessed by reviewing the spectral appearance (line width, signal-to-noise) of the creatine resonance obtained relative to concentrations (ratios-to-creatine) of choline, myo-inositol, and NAA.

RESULTS: Of 75 studies, 50 (66.6\%) were rated as readable: short TE $=17 / 50$ (34\%), long TE $=33 / 50$ (66\%), cephalic presentation in 36/50 (72\%) studies, breech in 10/50 (20\%) studies, and "other" presentation in 4/50 (8\%) studies (mean GA, 31.0 weeks). Twenty-eight of 50 fetuses (56\%) showed normal development (short TE $=12 / 28$, long TE $=16 / 28$ ), and 22/50 (44\%) showed pathology. Of the 75 studies, 25 (33.3\%) were not readable: short TE $=14 / 25(56 \%)$, long TE $=11 / 25$ (44\%), cephalic presentation in 20/25 (80\%) studies, breech in 4/25 (16\%) studies, and other presentation in 1 study (4\%) (mean GA, 30.1 week). Thirteen of 25 fetuses (52\%) showed normal development; 12/25 (48\%) showed pathology. Statistical analysis revealed no impact of the different parameters on the quality of spectra.

CONCLUSIONS: Single-voxel spectroscopy can be performed in approximately two-thirds of unsedated fetuses, regardless of the type of spectra, fetal presentation, GA, and pathology.

ABBREVIATIONS: FWHM = full width at half maximum; $G A=$ gestational age; $G W=$ gestational week; IUGR = intrauterine growth restriction; Lac $=$ lactate; Lip $=$ lipid; mlns = myo-inositol; PRESS = point-resolved spectroscopy

'H -MR spectroscopy has been established as a useful tool for understanding the pathophysiologic mechanisms of fetal brain injury. ${ }^{1-4}$ The intrinsic magnetic properties of the fetal brain are used to compile a spectrum by using the chemical-shift phenomenon. The spectrum contains different signal peaks based on identifiable metabolites. ${ }^{1,5}$ The composition of these metabolites may indicate abnormalities in the fetal brain metabolism, such as those that occur in developmental disorders; inborn errors of metabolism; and hypoxic encephalopathy ${ }^{6-10} \mathrm{MR}$ spectroscopy is a

Received December 27, 2011; accepted after revision April 27, 2012.

From the Departments of Radiology (V.B.-K., M.K., M.W., A.W., D.P.) and Anatomy and Cell Biology (P.C.B.), Medical University of Vienna, Vienna, Austria; and Depart ment of Gynecology and Obstetrics (D.P.), Medical University of British Columbia, BC Women's Hospital, Vancouver, British Columbia, Canada.

Please address correspondence to Vanessa Berger-Kulemann, MD, Department of Radiology, Medical University of Vienna/General Hospital, Waehringer Guertel 18-20, 1090 Vienna, Austria; e-mail: vanessa.berger-kulemann@meduniwien.ac.at http://dx.doi.org/10.3174/ajnr.A3196 complementary adjunctive tool to morphologic imaging of the brain. $^{1}$

However, the quality of the spectroscopic studies strongly depends on the homogeneity of the magnetic field and may be limited because of unrestricted fetal movement during the acquisition of the scan. Fetal sedation is recommended to avoid these motion artifacts. ${ }^{5,11,12}$ Nevertheless, on the basis of routine clinical experience, fetal sedation is usually not necessary. This may be primarily dependent on the gestational age and fetal head position. In the older and larger fetus, less movement in utero is possible. In the cephalic position, the fetal head may be more fixed in the maternal pelvis, preventing gross movement of the head.

The aim of this study was to assess the feasibility and quality of brain ${ }^{1} \mathrm{H}-\mathrm{MR}$ spectroscopy in unsedated fetuses and to evaluate whether the quality of the examination is dependent on the type of spectra (short/long TE), fetal presentation, GA, and/or fetal pathology. In addition, the results of MR spectroscopy were ana- 


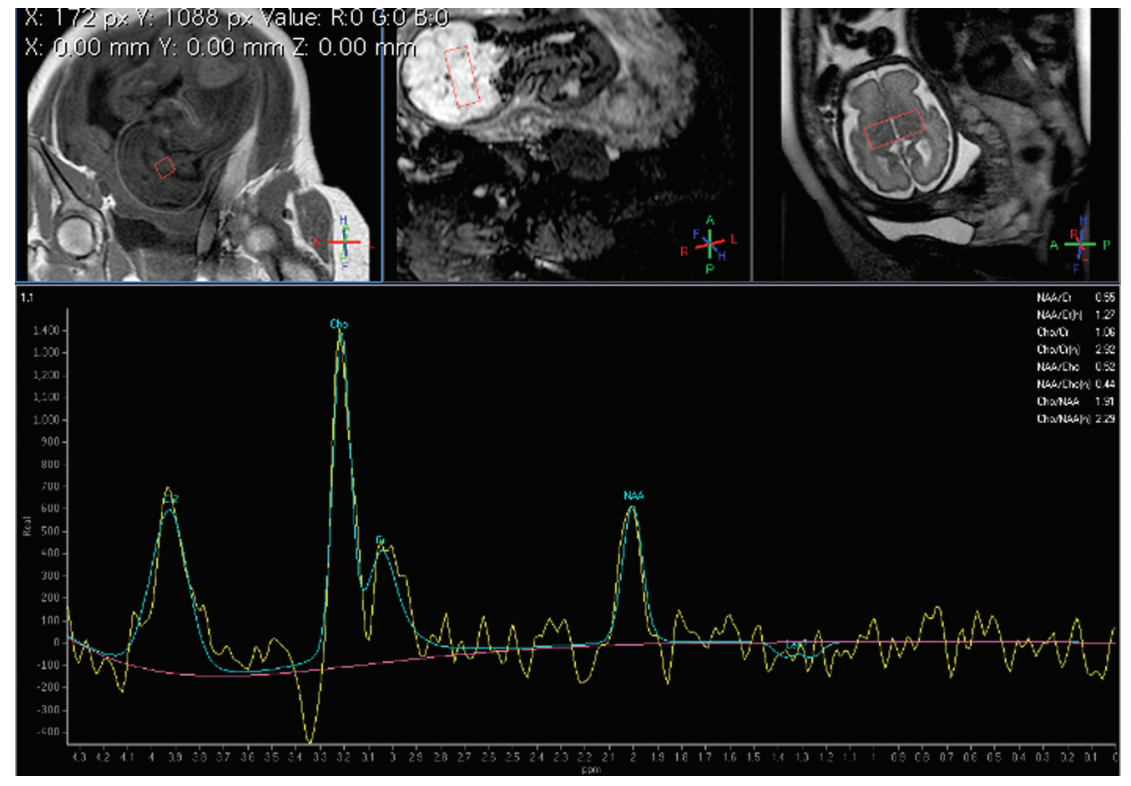

FIG 1. GW $29+6$, single-voxel MR spectroscopy of the brain. PRESS with a long TE (144 ms). VOI $2.7 \mathrm{~cm}^{3}$. Cephalic presentation. Normal age-related spectrum.

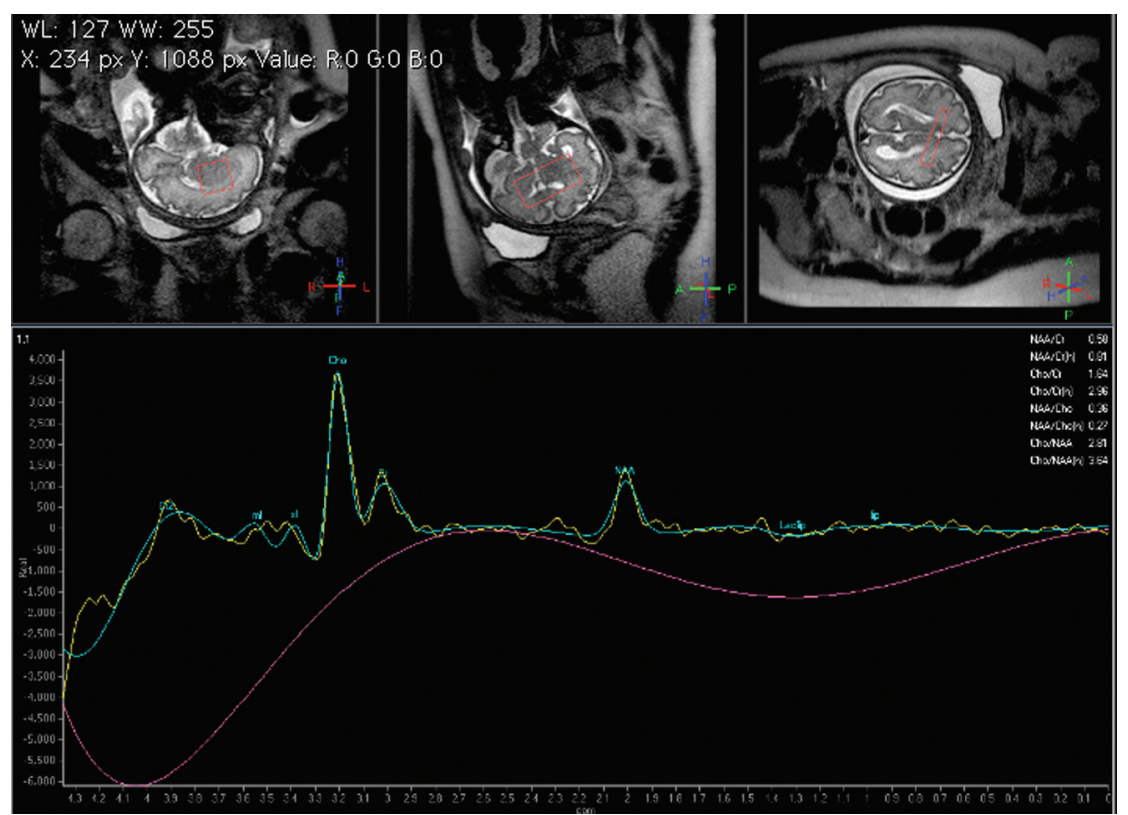

FIG 2. GW $31+3$, single-voxel MR spectroscopy of the brain. PRESS with a short TE $(35 \mathrm{~ms})$. VOI $2.7 \mathrm{~cm}^{3}$. Cephalic presentation. Normal age-related spectrum.

lyzed with regard to the changes in metabolite concentration with gestational age.

\section{MATERIALS AND METHODS}

This was a retrospective single-institution study; the local ethics committee granted approval. Within a 3-year 7-month period, a total of 75 single-voxel MR spectroscopic studies of the fetal brain in 70 singleton fetuses were performed at our institution. The patients underwent fetal MR imaging between gestational weeks 19 and 38 and were referred for MR imaging from the department of obstetrics and gynecology for further investigation of suspected brain abnormalities. Each pregnant woman gave written in- formed consent for the fetal MR imaging examination; no contraindication to the MR imaging examination was present. The women were scanned in the supine or left decubitus position. No premedication for sedation was given.

\section{MR Imaging and MR Spectroscopy}

Fetal MR imaging examinations were performed on a 1.5T scanner (Gyroscan; Philips Healthcare, Best, the Netherlands); imaging protocols depended on the region of interest and included at least T2-weighted TSE sequences in the coronal, sagittal, and axial planes.

In addition to morphologic imaging, an MR spectroscopy study was performed in each patient. MR spectroscopy was performed in 2 groups with presumed normal brain development for study purposes. ${ }^{1}$ There, both long and short TEs were the goal. In addition, MR spectroscopy was performed when there were brain pathologies, such as tumors, and in cases with suspected metabolic changes in the fetal brain, for instance, in IUGR.

Because a radiologist was present at the examination, the studies were actively monitored. A sensitivity-encoding cardiac coil and PRESS (spin-echo) with a long TE (144 ms) and a short TE (35 ms) were used. The chosen voxel size was as small as possible and ranged from $2.7(14 \times 14 \times$ $14 \mathrm{~mm})$ to $5.6 \mathrm{~cm}^{3}(20 \times 20 \times 14 \mathrm{~mm})$, depending on gestational age.

To investigate normally developing white matter, we located the VOI within the cerebral hemisphere at the level of the centrum semiovale. As a consequence of the small size of the fetal brain with respect to the VOI, the basal ganglia region was included in most cases. In abnormal brains, the VOI was located in the area of visible pathology after GW 28. In younger fetuses, the voxel contained future gray matter and future white matter due to the characteristic lamination in these maturational stages of pregnancy. ${ }^{13}$

At each TE, sequences were obtained with water saturation. The number of acquisitions for 1 spectrum was 128 . Scanning time for the MR spectroscopy data acquisition, resulting from the number of acquisitions, TR of 2 seconds, and prescan adjustments, was a maximum of 5 minutes. The presence of gross movement could be excluded because a scout view was obtained after the MR spectroscopy sequence to confirm the constant position of the region of interest. If the sequence needed to be repeated due to movement, it was repeated once; if this spectrum was not readable, it was not repeated again. 
Overall, we obtained 75 spectra: 44 with long TEs and 31 with short TEs. In 5 fetuses, spectra with short and long TEs were obtained; in 6 fetuses, the MR spectroscopy studies with a short TE had to be repeated to achieve acceptable quality. Data were processed with the use of SpectroView within the Extended MR Workspace 2.6.3 (Philips Healthcare) and consisted of exponential filtering, automatic phase correction, and initial baseline subtraction. For the line-fitting of the spectra signals of mIns (short TE only), Cho, Cr, NAA, and glutamine/glutamate (short TE only) peaks, optional Lip and Lac were included.

\section{Spectra Analysis}

The MR spectroscopy studies were retrospectively evaluated on a PACS by 1 experienced radiologist and 1 physicist, who specialized in MR spectroscopy. They were blinded to the diagnosis. The type of spectra, the fetal presentation, GA, and type of pathology (if present) were recorded. Fetal presentation was defined by the position of the fetus with respect to the pelvic inlet: cephalic presentation, breech presentation, or "other" presentation.

The 2 readers evaluated the quality of the spectra, judging the presence and splitting of choline (3.2 ppm) and creatine (3.05 ppm) resonance lines separately, and then compared the results. As a secondary spectra analysis, we reviewed the Cho, mIns, and NAA relative concentrations (ratiosto-creatine). For this purpose, we split the data on the basis of normal fetal development and MR spectroscopy sequence used (long-versus-short TEs).

We also evaluated the presence of lactate signals, defined as an upright (short TE) or an inverted (long TE) doublepeak, appearing at $1.3 \mathrm{ppm}$, in readable spectra with short and long TEs in fetuses with and without pathology.

\section{Statistical Analysis}

All calculations were performed by using the Statistical Package for the Social Sciences, Version 17.0 (SPSS, Chicago, Illinois). The impact of the type of spectra, fetal position, GA, and fetal pathologies on readability was assessed by using crosstabs and Fisher exact or Fisher-Freeman-Halton tests for each variable separately as a logistic regression to model all possible impact factors simultaneously.

In addition, we performed statistical analysis of the 2 groups (readable/nonreadable), which involved the comparison of

FWHM of the Cr line, as assessed by automatic postprocessing on the scanner platform and given in parts per million. To compare the 2 groups with respect to GA and FWHM, an unpaired Student $t$ test was used. Correlations between GA and metabolite concentrations were assessed by using the Pearson correlation coefficient and linear regression analysis.

A $P$ value $\leq .05$ was considered to indicate significant results. 
Table 1: Fetal pathologies of readable and nonreadable spectra with short TEs

\begin{tabular}{l} 
Short TEs (35 ms) \\
\hline Readable \\
Zellweger syndrome \\
Solitary median maxillary central incisor \\
Infratentorial neoplasm \\
IUGR \\
Abdominal mass \\
Cerebral edema \\
Congenital diaphragmatic hernia \\
Microphthalmos \\
Gastroschisis \\
Nonreadable \\
Ovarian cyst \\
Congenital cystic adenomatoid malformation \\
Abdominal mass
\end{tabular}

\begin{tabular}{l}
$\begin{array}{l}\text { Table 2: Fetal pathologies of readable and nonreadable spectra } \\
\text { with long TEs }\end{array}$ \\
\hline \multicolumn{1}{c}{ Long TEs (144 $\mathbf{~ m s})$} \\
\hline Readable \\
Corpus callosum agenesia \\
Infratentorial mass \\
IUGR \\
Chiari II malformation \\
Asymmetric ventricles \\
Cerebral ischemia \\
Bilateral cheilognathopalatoschisis \\
Cerebral arteriovenous fistula \\
Lung hypoplasia \\
Kidney agenesia \\
Intracranial teratoma \\
Nonreadable \\
Vitium cordis \\
Congenital diaphragmatic hernia
\end{tabular}

Due to the relatively small sample size, no multiplicity corrections were performed to avoid an increasing error of the second type.

\section{RESULTS}

Of 75 studies, $50(66.6 \%)$ were rated as readable (Figs 1-4): short $\mathrm{TE}=17 / 50(34 \%)$, long $\mathrm{TE}=33 / 50(66 \%)$. Cephalic presentation was found in $36 / 50$ (72\%) studies, breech in 10/50 (20\%) studies, and other presentation in 4/50 (8\%) studies (mean gestational age, $31.0 \mathrm{GWs}$ ). There were $28 / 50$ fetuses (56\%) who showed normal development ( hort $\mathrm{TE}=12 / 28$, long $\mathrm{TE}=16$ / 28) (Figs 1, 2, and 4) and 22/50 (44\%) who showed any kind of pathology (Fig 3).

Of the 75 studies, 25 (33.3\%) were nonreadable: short $\mathrm{TE}=$ $14 / 25(56 \%)$, long TE $=11 / 25(44 \%)$. Cephalic presentation was observed in 20/25 (80\%) studies; breech presentation, in 4/25 (16\%) studies; and other presentation, in 1 study (4\%) (mean gestational age, $30.1 \mathrm{GWs})$. There were $13 / 25$ fetuses (52\%) that showed normal development and 12/25 (48\%) that showed any kind of pathology. All pathologies in the different groups are summarized in Tables 1 and 2.

Nonreadable spectra were attributable to fetal movement in approximately $75 \%$ of cases and to an unfavorable position of the region of interest within the magnetic field in approximately $25 \%$ of the cases. In $69 / 75$ spectra, the voxel was located at the level of the centrum semiovale, including the basal ganglia region in most cases. In 6/75 spectra, the voxel was located in the region of visible brain pathology; all these spectra were readable.

Neither univariate nor multivariate analysis showed any impact of the different parameters on the quality of the spectra (type of spectra, $P=.085$; cephalic presentation, $P=.758$; GA, $P=$ .316; pathology, $P=.805$ ). No statistical significance was found.

GA for readable and nonreadable groups did not differ (31.0 \pm 4.66 versus $30.1 \pm 4.99$ weeks, $P=.444$ ), and both groups contained a similar relative abundance of normal-versus-pathologically-developing fetuses (readable, 56\% normal; nonreadable, $52 \%$ normal).

The analysis of the FWHM of the Cr line for the readable group $(n=50)$ yielded an $\mathrm{FWHM}_{\mathrm{Cr}}$ of $0.098 \pm 0.057 \mathrm{ppm}$ and, for the nonreadable group $(n=25)$, an $\mathrm{FWHM}_{\mathrm{Cr}}$ of $2.42 \pm 3.65$ $\operatorname{ppm}(P<.001)$.

The correlation analysis showed a linear increase in NAA/Cr ratio $(r=0.605, P=.037)$ and a decrease in $\mathrm{Cho} / \mathrm{Cr}(r=0.258$, $P=.419)$ and $\mathrm{mIns} / \mathrm{Cr}$ ratios $(r=0.514, P=.088)$ with GA in the readable short TE spectra of normally developed fetuses (Fig $5 A-C)$ as well as an increase in the NAA/Cr ratio $(r=0.206, P=$ $.499)$ and a decrease in the $\mathrm{Cho} / \mathrm{Cr}$ ratio $(r=0.447, P=.083)$ with GA in the readable long TE spectra of normally developed fetuses (Fig $5 D,-E$ ). We found a statistically significant value for NAA/Cr in short TEs. However, in all other cases, the correlation did not reach significant values.

An upright (short TE) or inverted (long TE) Lac signal was observed in several readable spectra, respectively, in fetuses with and without pathology (Fig 6). However, in some spectra with long TEs, a distinct double-peak Lac signal was detectable, while other spectra showed an indistinct signal in the Lac region, potentially representing Lac. In readable spectra with short TEs, a large Lip signal was found in almost half the spectra, and a clear differentiation between Lip and Lac was not possible (Figs 4 and 6). The Fisher exact test showed no statistically significant correlation between the presence of Lac and fetal pathology $(P=.201)$. However, a tendency toward the presence of Lac in pathologic fetuses at short TEs could be detected $(P=.081)$.

\section{DISCUSSION}

Prenatal MR spectroscopy of the brain is feasible in most unsedated fetuses, regardless of the type of spectra, fetal presentation, GA, or fetal pathology. Nevertheless, approximately one-third of MR spectroscopy studies in unsedated fetuses are of limited quality.

The quality of a spectroscopic study depends on the homogeneity of the magnetic field and restricted movement of the sample. In addition, for an optimal signal-to-noise ratio, the VOI should be as close to the receiver coil as possible. ${ }^{1}$ The use of flexible coil arrays allows comfortable placement of the receiver coils and the choice of the best-positioned array element for signal detection. ${ }^{1}$

The important limiting factor for the quality of MR spectroscopy studies is the presence of motion-associated artifacts. While gross motion of the fetal head usually leads to an unreadable spectrum, the intrinsic motion composed of maternal breathing movements, vascular flow, and amniotic fluid waves may impair the quality of the examination. In case of unrestricted fetal move- 
$\mathrm{TE}=\mathbf{3 5} \mathrm{ms}$

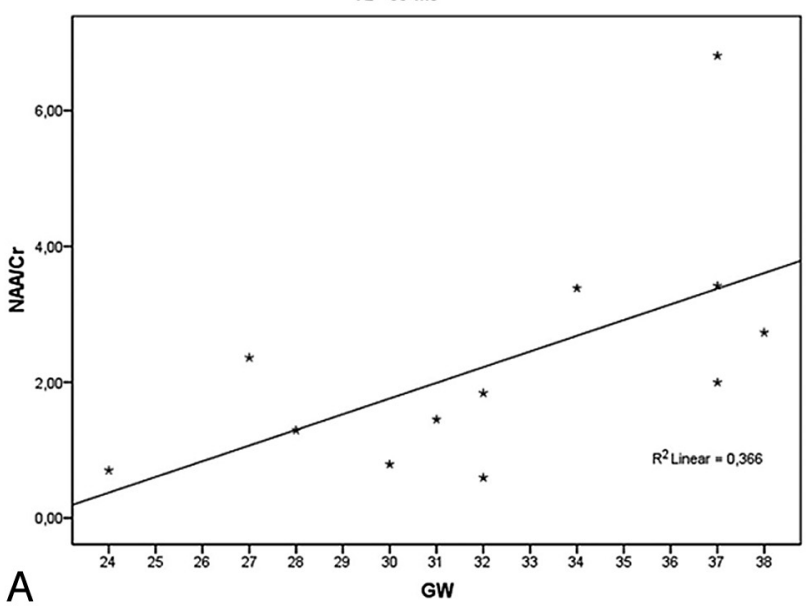

$\mathrm{TE}=35 \mathrm{~ms}$

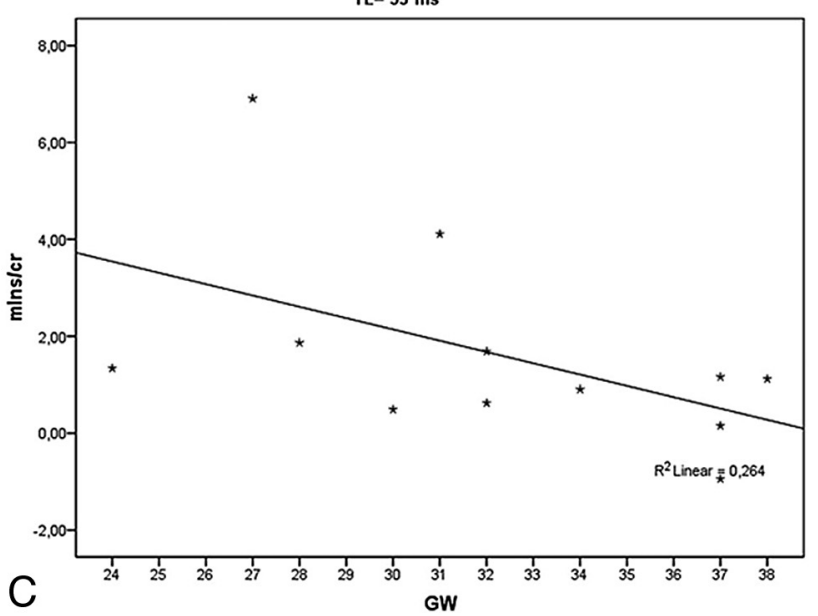

GW

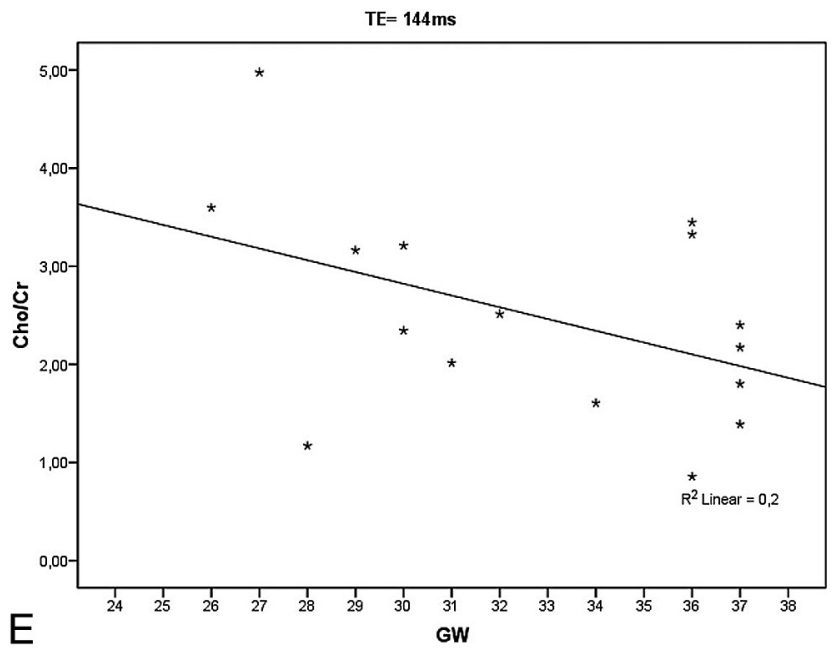

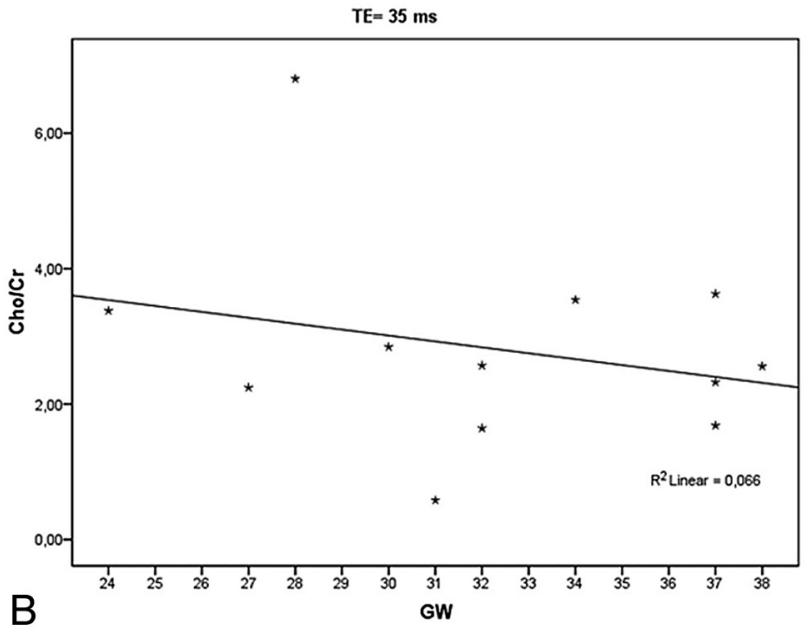

$T E=144 \mathrm{~ms}$

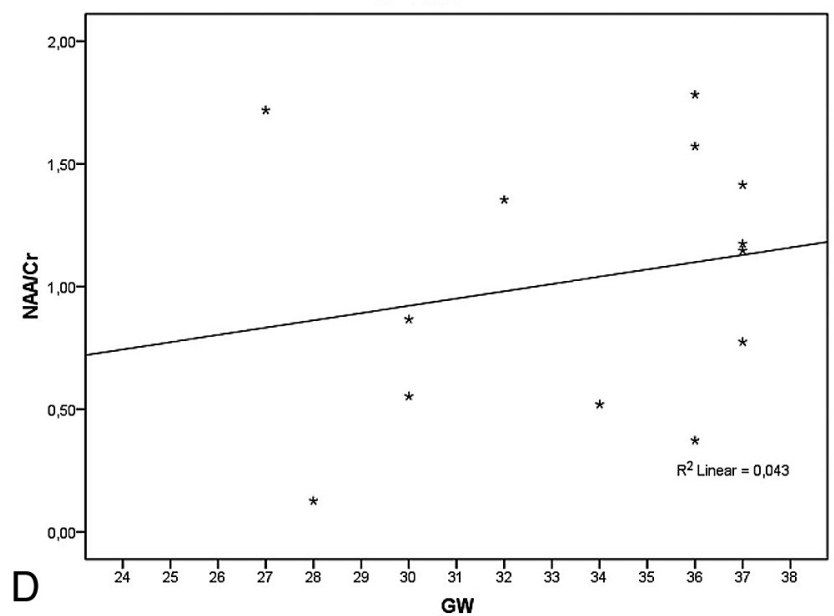

FIG 5. $A-C$, The linear correlation analysis confirmed the significant increase of NAA/Cr ratio and shows a not significant decrease of $C$ ho/Cr and mlns/Cr ratios with GA in the readable short TE spectra of normally developed fetuses. $D$ and $E$, Note the increase of the NAA/Cr ratio and the decrease of the $\mathrm{Cho/Cr}$ ratio with GA in the readable long TE spectra of normally developed fetuses.

ments, scout images may have to be repeated after spectroscopy to ensure the placement of the VOI in the chosen region. ${ }^{1}$ Although special time-consuming shimming procedures would be helpful to increase MR spectroscopy quality, the effect of fetal movements is more pronounced with longer shimming/acquisition times. A reduction of shimming/acquisition time is helpful to avoid motion-associated artifacts, even at the cost of lower magnetic field homogeneity. Other parameters, such as fetal presentation, GA, 


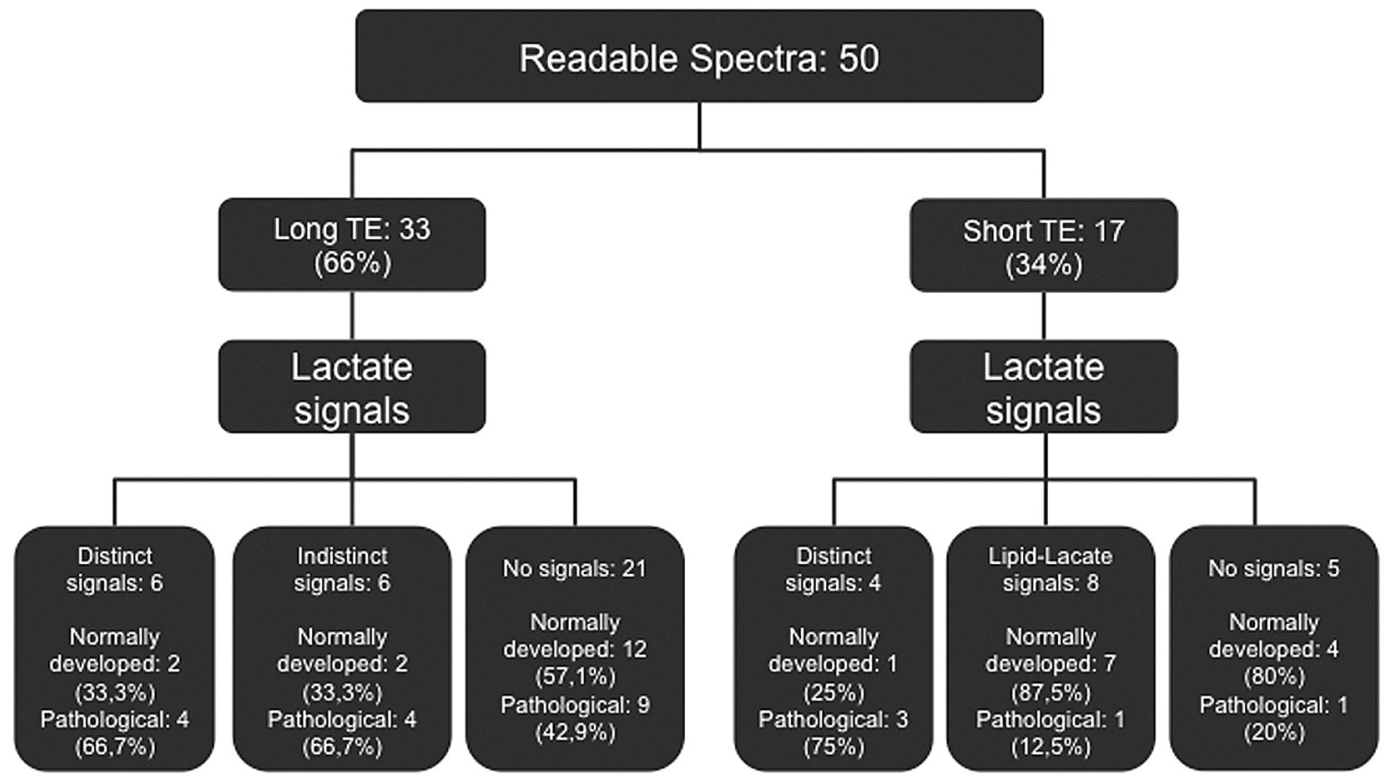

FIG 6. Lactate signals found in readable spectra with long and short TEs of normally and pathologically developed fetuses.

and pathology, may influence the occurrence of movements. Small fetuses at a younger gestational age have more amniotic fluid and room in utero, compared with fetuses at a later gestational age and of a larger size, where gross movements are more likely. In the cephalic presentation, it would seem that the fetal head is fixed in the maternal pelvis, where movements are less possible than in the breech or other presentations. ${ }^{14}$ However, this was not proved to be true; no correlation was found between spectra quality and fetal presentation or GA.

To avoid motion-associated artifacts, fetal sedation is recommended. ${ }^{10-12}$ Maternal premedication with 10-mg diazepam (benzodiazepine) is considered to be a safe method for both mother and fetus. ${ }^{9,12}$ Brunel et al $^{11}$ suggest the oral administration of flunitrazepam, a short- to intermediate-acting benzodiazepine, 15 minutes to 1 hour before the examination. ${ }^{15-17}$ However, even with a single maternal dose of a benzodiazepine, the mother must be monitored after the MR imaging examination. Adverse effects, such as impairment of cognitive functions, with a lack of concentration and confusion, are possible (hangover-like effect). ${ }^{18}$ Impaired psychomotor functions affect reaction times and driving skills. Other adverse effects include gastrointestinal disturbances and vomiting, and respiratory depression may occur in case of overdose. ${ }^{18}$ Fetal behavior, which can be visualized by dynamic MR imaging, ${ }^{19}$ cannot be evaluated reliably in sedated fetuses. This may impair the "neurologic" assessment of the fetus, which consists of morphologic, metabolic, and movement evaluation. $^{20}$

Partial volume effects can hamper the interpretation of the prenatal MR spectra. Relatively large VOIs cannot be placed in morphologically homogeneous prenatal brain structures. Although the small size of the fetal brain makes it impossible, in most cases, to exclude CSF spaces from the VOI, the inclusion of bony structures of the calvaria or skull base must be avoided to prevent lipid contamination of short TE spectra.

The fact that almost three-quarters of the long-TE studies were readable versus only $17 / 31$ of the short-TE studies, though not statistically significant, might be explained by several factors. Short-TE spectra have more complicated baselines. In combination with the more complicated spectral pattern and possible lipid contamination, short-TE spectra are also definitely more prone to be unreadable. We recommend measuring the long-TE spectra first, and if the quality is satisfactory, then the short TE spectra can be measured. Additional short TE spectra might be useful, for instance, to evaluate mIns in case of suspected gliosis.

The changing anatomy of the fetal brain during maturation is also challenging. Gyration of the gray matter starts at 18 weeks' GA; until 35 weeks, the entire primary and most of the secondary sulci are present. ${ }^{19} \mathrm{Up}$ to approximately 30 weeks, the brain parenchyma differs markedly from the postnatal stages. ${ }^{13}$ In contrast to defined regions of gray and white matter that can be delineated postnatally, a laminated appearance, consisting of layers with different cell attenuation contents and intra-/extracellular water, dominates the immature fetal brain. Consequently, a voxel of the size described above will contain various cell compounds, and the resulting spectrum will reflect the average metabolic output of histologically different structures.

In addition, mature myelin is almost absent intrauterinely because cerebral myelination is mostly a postnatal process. ${ }^{19,21} \mathrm{Pr}-$ emyelination may be recognized by using diffusion-weighted images, as early as gestational week $20 .^{14,19,22}$ Definite metabolic characteristics of unmyelinated/premyelinated white matter are unknown, but several in vitro anatomic and metabolic findings and the results of in vivo studies suggest that the premyelination processes and ongoing myelination are reflected by NAA. ${ }^{23-29}$ However, the impact of premyelinating structures on the spectroscopy signals is probably too weak during the second trimester of pregnancy to redound a visible amount to the composition of the spectrum.

We have analyzed the results of MR spectroscopy on the expected increase of NAA/Cr ratios and decrease of Cho/Cr ratios ${ }^{26}$ as well as the mIns/Cr ratio. The NAA/Cr ratio reached significant results; the Pearson correlation coefficient showed the relevant 
impact of the GA on the ratio. However, the correlation did not reach significant values in most cases, most probably due to the small number of readable spectra for long and short TEs for normally developed fetuses. The Cho/Cr ratio in the fetal period seems to be associated with the GA. Girard et $\mathrm{al}^{27}$ found a significant decrease in Cho with increasing GA between GWs 22 and 39. Kok et $\mathrm{al}^{26}$ described no statistically significant decrease in Cho in fetuses from GW 30 upward, but there was a decrease in the Cho/Cr ratio, confirmed by Story et al. ${ }^{30}$

The evaluation of brain Lac was complicated by indistinct signals in the Lip/Lac region. TEs of 35 and $144 \mathrm{~ms}$ are not optimal for the detection of Lac because the sensitivity to Lip contamination is increased in short-TE spectra and the Lac signal is suppressed by spin-spin relaxation in longer TE spectra. ${ }^{31}$ The Lip signal rises to a peak at $1.3 \mathrm{ppm}$ and may mask a Lac peak, ${ }^{31}$ as found in several spectra with short TEs (Fig 6). The presence of fetal brain Lac is associated with brain pathology and hypoxia and IUGR. ${ }^{30-33}$ A Lac signal was found in most fetuses with IUGR. ${ }^{31}$ However, growth-restricted fetuses with a good short-term outcome and normally developed fetuses also showed brain Lac (Fig $6),{ }^{30}$ suggesting that Lac is an important source of energy for the normally developing brain. ${ }^{34}$ Differentiation between normal and abnormal brain development by means of only a Lac signal is currently not possible. Further studies to investigate brain Lac in normally and abnormally developing fetuses and larger study populations are needed to establish a standard of reference.

Due to the retrospective design, the study population was inhomogeneous, comprising, for study purposes, MR spectroscopy with presumed normal spectra and studies of patients with brain pathology and suspected metabolic changes. Variable imaging protocols with different voxel sizes, located in the region of interest, were included. Prospective studies that avoid these limitations are required to confirm our findings.

\section{CONCLUSIONS}

Single-voxel MR spectroscopy can be performed in approximately two-thirds of unsedated fetuses after gestational week 19, regardless of the type of spectra, fetal presentation, GA, or pathology.

Disclosures: Daniela Prayer—RELATED: Grant: Jubiläumsfonds projekt 12960. Money paid to the institution.

\section{REFERENCES}

1. Pugash D, Krssak M, Kulemann V, et al. Magnetic resonance spectroscopy of the fetal brain. Prenat Diagn 2009;29:434-41

2. Huppi PS, Amato M. Advanced magnetic resonance imaging techniques in perinatal brain injury. Biol Neonate 2001;80:7-14

3. Toft PB, Leth H, Lou HC, et al. Metabolite concentrations in the developing brain estimated with proton MR spectroscopy. J Magn Reson Imaging 1994;4:674-80

4. Cady EB, Amess P, Penrice J, et al. Early cerebral-metabolite quantification in perinatal hypoxic-ischaemic encephalopathy by proton and phosphorus magnetic resonance spectroscopy. Magn Reson Imaging 1997;15:605-11

5. Girard N, Gouny SC, Viola A, et al. Assessment of normal fetal brain maturation in utero by proton magnetic resonance spectroscopy. Magn Reson Med 2006;56:768-75

6. Kok RD, Steegers-Theunissen RP, Eskes TK, et al. Decreased relative brain tissue levels of inositol in fetal hydrocephalus. Am J Obstet Gynecol 2003;188:978-80

7. Azpurua H, Alvarado A, Mayobre F, et al. Metabolic assessment of the brain using proton magnetic resonance spectroscopy in a growth-restricted human fetus: case report. Am J Perinatol 2008;25:305-09

8. Astley SJ, Richards T, Aylward EH, et al. Magnetic resonance spectroscopy outcomes from a comprehensive magnetic resonance study of children with fetal alcohol spectrum disorders. Magn Reson Imaging 2009;27:760-78

9. Roelants-van Rijn AM, Groenendaal F, Stoutenbeek P, et al. Lactate in the foetal brain: detection and implications. Acta Paediatr 2004;93:937-40

10. Roelants-Van Rijn AM, van der Grond J, de Vries LS, et al. Value of ${ }^{1} \mathrm{H}$-MRS using different echo times in neonates with cerebral hypoxia-ischemia. Pediatr Res 2001;49:356-62

11. Brunel H, Girard N, Confort-Gouny S, et al. Fetal brain injury. JNeuroradiol 2004;31:123-37

12. Gerards FA, Stoutenbeek P, Gooskens RH, et al. Diagnostic value of prenatal MRI in fetus with intracranial anomalies diagnosed by ultrasonography [in Dutch]. Ned Tijdschr Geneeskd 2001;145:179-84

13. Kostović I, Judas M, Rados M, et al. Laminar organization of the human fetal cerebrum revealed by histochemical markers and magnetic resonance imaging. Cereb Cortex 2002;12:536-44

14. Kasprian G, Brugger PC, Weber M, et al. In utero tractography of fetal white matter development. Neuroimage 2008;43:213-24

15. Kanto J, Erkkola R, Kangas L, et al. Placental transfer of flunitrazepam following intramuscular administration during labour. $\mathrm{Br} J$ Clin Pharmacol 1987;23:491-94

16. Oelschläger H. Chemical and pharmacologic aspects of benzodiazepines [in German]. Schweiz Rundsch Med Prax 1989:78:766-72

17. Kanto JH. Use of benzodiazepines during pregnancy, labour and lactation, with particular reference to pharmacokinetic considerations. Drugs 1982:23:354-80

18. Hindmarch I. A repeated dose comparison of three benzodiazepine derivative (nitrazepam, flurazepam and flunitrazepam) on subjective appraisals of sleep and measures of psychomotor performance the morning following night-time medication. Acta Psychiatr Scand 1977:56: 373-81

19. Prayer D, Kasprian G, Krampl E, et al. MRI of normal fetal brain development. Eur J Radiol 2006;57:199-216

20. Legido A, Valencia I, Smith JD. Fetal neurological evaluation [in Spanish]. Rev Neurol 2004:15;39:454-64

21. Counsell SJ, Maalouf EF, Fletcher AM, et al. MR imaging assessment of myelination in the very preterm brain. AJNR Am J Neuroradiol 2002;23:872-81

22. Huang $\mathrm{H}$, Zhang J, Wakana $\mathrm{S}$, et al. White and gray matter development in human fetal, newborn and pediatric brains. Neuroimage 2006:15;33:27-38

23. Madhavarao CN, Hammer JA, Quarles RH, et al. A radiometric assay for aspartoacylase activity in cultured oligodendrocytes. Anal Biochem 2002;308:314-19

24. Kirmani BF, Jacobowitz DM, Namboodiri MA. Developmental increase of aspartoacylase in oligodendrocytes parallels CNS myelination. Brain Res Dev Brain Res 2003;140:105-15

25. Kato T, Nishina M, Matsushita $K$, et al. Neuronal maturation and $\mathrm{N}$-acetyl-L-aspartic acid development in human fetal and child brains. Brain Dev 1997;19:131-33

26. Kok RD, van den Berg PP, van den Bergh AJ, et al. Maturation of the human fetal brain as observed by $1 \mathrm{H}$ MR spectroscopy. Magn Reson Med 2002;48:611-16

27. Girard N, Fogliarini C, Viola A, et al. MRS of normal and impaired fetal brain development. Eur J Radiol 2006;57:217-25

28. Heerschap A, Kok RD, van den Berg PP. Antenatal proton MR spectroscopy of the human brain in vivo. Childs Nerv Syst 2003; 19:418-21

29. Kreis R, Hofmann L, Kuhlmann B, et al. Brain metabolite composi- 
tion during early human brain development as measured by quantitative in vivo $1 \mathrm{H}$ magnetic resonance spectroscopy. Magn Reson Med 2002;48:949-58

30. Story L, Damodaram MS, Allsop JM, et al. Brain metabolism in fetal intrauterine growth restriction: a proton magnetic resonance spectroscopy study. Am J Obstet Gynecol 2011;205:483.e1-8

31. Charles-Edwards GD, Jan W, To M, et al. Non-invasive detection and quantification of human foetal brain lactate in utero by magnetic resonance spectroscopy. Prenat Diagn 2010;30:260 -66
32. Wolfberg AJ, Robinson JN, Mulkern R, et al. Identification of cerebral lactate using magnetic resonance spectroscopy. Am J Obstet Gynecol 2007;196:e9-11

33. Moxon-Lester L, Sinclair K, Burke C, et al. Increased cerebral lactate during hypoxia may be neuroprotective in newborn piglets with intrauterine growth restriction. Brain Res 2007;1179:79-88

34. Taberno A, Vicario C, Medina JM. Lactate spares glucose as a metabolic fuel in neurons and astrocytes from primary culture. Neurosci Res1996;26:369-76 